\title{
Práticas de educação em saúde na escola: prevenção às parasitoses entre crianças do ensino fundamental em uma cidade no interior do Pará
}

\author{
Health education practices at school: preventing parasitic infections among elementary \\ school children in a city in the interior of Pará
}
Prácticas de educación para la salud en la escuela: prevención de infecciones parasitarias en niños de escuela primaria en una ciudad del interior de Pará

Evelyn de Sousa Moreira ${ }^{1 *}$, Pedro Henrique Gomes Vieira ${ }^{1}$, Daniele Regina da Silva Fernandes ${ }^{1}$.

\section{RESUMO}

Objetivo: Promover educação em saúde para crianças em idade escolar em uma cidade no interior do Pará. Relato de experiência: Atividades de educação em saúde foram realizadas com alunos do $3^{\circ}$ ao $5^{\circ}$ do ensino fundamental de uma escola pública de uma cidade no interior do Pará. Como recurso audiovisual foi utilizado um vídeo, disponível no Youtube e chamado "Doutor Sabão contra as parasitoses", que trata sobre a prevenção e promoção de parasitoses além de higiene pessoal básica. Em seguida uma roda de conversa era formada e os participantes eram informados sobre os principais parasitos de importância médica e aprendiam como se prevenir das doenças causadas por eles. Ao final uma dinâmica sobre higienização correta das mãos era realizada com as crianças fazendo uso de álcool em gel. Considerações Finais: A extensão permite que a universidade se aproxime da população e que possa prestar serviços que visam melhorar a qualidade de vida das pessoas. Esse projeto possibilitou a interação com essas crianças que muitas vezes não tem a oportunidade de obter informações com tanta frequência haja vista que os professores têm uma sobrecarga de demandas. Ações como essa precisam ser permanentes para que assim o conhecimento possa ser disseminado.

Palavras-chave: Parasitoses, Educação em saúde, Higienização das mãos.

\begin{abstract}
Objective: To promote health education for school-age children in a city in the state of Pará. Experience report: Health education activities were carried out with students from the 3rd to the 5th of elementary school in a public school in a city in the interior of Pará. As an audiovisual resource, a video was used, available on Youtube and called "Doutor Sabão contra parasitoses", which deals with the prevention and promotion of parasites in addition to basic personal hygiene. Then a conversation circle was formed and the participants were informed about the main parasites of medical importance and learned how to prevent diseases caused by them. At the end, a dynamic on correct hand hygiene was performed with the children using alcohol gel. Final Considerations: The extension allows the university to get closer to the population and to provide services aimed at improving people's quality of life. This project made it possible to interact with these children, who often do not have the opportunity to obtain information so often given that teachers have an overload of demands. Actions like this need to be permanent so that knowledge can be disseminated.
\end{abstract}

Keywords: Parasitosis, Health education, Hand hygiene.

1 Universidade Federal do Pará (UFPA), Altamira - PA. *E-mail: danirsfernandes@gmail.com

SUBMETIDO EM: 8/2020

ACEITO EM: 9/2020

PUBLICADO EM: 2/2021 


\section{RESUMEN}

Objetivo: Promover la educación en salud para niños en edad escolar en una ciudad del estado de Pará. Informe de experiencia: Se llevaron a cabo actividades de educación en salud con estudiantes de $3^{\circ}$ a $5^{\circ}$ de primaria en una escuela pública de una ciudad del interior de Pará. Como recurso audiovisual se utilizó un video, disponible en Youtube y denominado "Doutor Sabão contra parasitosis", que trata sobre la prevención y promoción de parásitos además de la higiene personal básica. Luego se formó un círculo de conversación y los participantes fueron informados sobre los principales parásitos de importancia médica y aprendieron cómo prevenir las enfermedades causadas por ellos. Al final, se realizó una dinámica de correcta higiene de manos con los niños utilizando gel de alcohol. Consideraciones finales: La extensión permite a la universidad acercarse a la población y brindar servicios orientados a mejorar la calidad de vida de las personas. Este proyecto permitió interactuar con estos niños, que muchas veces no tienen la oportunidad de obtener información con tanta frecuencia dado que los profesores tienen una sobrecarga de demandas. Acciones como esta deben ser permanentes para que se pueda difundir el conocimiento.

Palabras clave: Parasitosis, Educación sanitaria, Higiene de manos.

\section{INTRODUÇÃO}

O enteroparasitisimo é o estado de infecção em que a agressão repercute prejudicialmente sobre o hospedeiro e é causado principalmente por protozoários e helmintos sendo muito recorrente na África, Ásia e América Latina especialmente em regiões periféricas e favelas atingindo todas as faixas etárias e tendo como sintomas principais: diarreia, anemia, colite, desnutrição, interferência no desenvolvimento cognitivo e físico (no caso de crianças e jovens), alterações psicossociais e até óbitos (MAIA CVA, et al., 2015).

As parasitasses são um problema de saúde pública muito frequente e são consideradas como indicadores de desenvolvimento econômico de um país visto que afetam, principalmente, indivíduos jovens e desencadeiam problemas intestinais além baixo rendimento corporal e atraso no desenvolvimento escolar (DIAS DS, et al., 2013). Para que esse problema possa ser solucionado ações de orientação sobre prevenção e tratamento devem ser realizadas a fim de que a população possa ser educada evitando danos à saúde infantil decorrentes da falta de conhecimento acerca dessas doenças (MONTEIRO AMC, et al., 2009).

Os estados mais pobres tendem a apresentar maior prevalência de parasitoses pois não possuem condições sanitárias adequadas à população principalmente nas periferias das cidades e de acordo com o Sistema Nacional de Informações sobre Saneamento (SNIS), 50,26\% (aproximadamente 102 milhões de pessoas) da população brasileira padece com o precário acesso à coleta de esgoto, sendo que na América Latina, pesquisas apontam que a média de infecções varia entre $30 \%$ e $53 \%$ e que aproximadamente 46 milhões de crianças entre 1 e 14 anos corre o risco de serem contaminadas por algum parasito (BRAGAGNOLLO GR, et al., 2019).

No Brasil, as parasitoses representam um grave problema de saúde pública pois a maior parte de sua população vive em condições financeiras muito difíceis e ainda com falta de água tratada, condições precárias de higiene pessoal, sem saneamento básico aliadas a uma condição climática e ambiental desfavorável que promove a proliferação de parasitos além da falta de escolaridade (SOUSA FCA, et al., 2019).

Projetos relacionados à prevenção de parasitoses tem a intenção de sensibilizar a população a adquirir hábitos saudáveis de higiene alimentar, hídricos e ambientais, ou seja, garante educação social a partir do conhecimento pois permite ao cidadão conhecer e identificar as doenças e assim garantir sua saúde (DIAS DS, et al., 2013).

Muito embora Brasil tenha passado por modificações que melhoraram a qualidade de vida de sua população nas últimas décadas, as parasitoses intestinais seguem endêmicas em diversas áreas do país representando um grave problema de saúde pública que acomete um grande número de crianças, podendo ocasionar em graves prejuízos ao seu desenvolvimento físico e escolar além de representarem a porta de entrada para outras doenças (BELO VS, et al., 2012; HANEMANN T, et al., 2015). 
O desenvolvimento de parasitoses, na maioria das vezes, é decorrente do meio e das condições as quais as pessoas estão expostas e acomete principalmente a população que apresenta déficit em determinantes sociais tais como: educação, abastecimento de água e saneamento, além de condições precárias de habitação e hábitos de higiene pessoal e de alimentos inadequados (SILVA NETO AP, et al., 2013).

As crianças contribuem sobremaneira para a disseminação de doenças visto que tem hábitos difíceis de serem controlados e que só favorecem a disseminação de parasitoses: levar as mãos e objetos à boca, falta de prática para lavar as mãos e de outros hábitos de higiene, proximidade física constante com adultos e um sistema imune ainda precário se levarmos em conta os com idade pré-escolar que não apresentam imunidade para agentes infecciosos mais comuns pois não tiveram exposição prévia (NESTI MM e GOLDBAUM M, 2007).

De acordo com a Organização Mundial de Saúde (OMS) a estratégia de promoção à saúde é fundamental para o estabelecimento de políticas públicas que desenvolvem e favorecem as habilidades pessoais e coletivas, contribuindo para melhorar a qualidade de vida e de saúde (SILVA PMC, et al., 2013).

Uma forma de prevenir parasitoses em crianças são ações educativas que visam o aprendizado. Na escola as crianças estão aptas a se envolverem em atividades lúdicas nas quais o conhecimento se constrói coletivamente (NOVAES AKB, et al., 2017).

O ensino de parasitologia abriga o estudo de diversas doenças parasitárias que acometem o ser humano. Por este motivo é tão importante que o tema seja abordado dentro de sala de aula visando a prevenção dessas doenças e auxiliando os alunos a praticarem normas de higiene eficazes contra essas enfermidades. Para tanto é preciso fazer uso da educação em saúde que é entendida como uma forma de ensino e aprendizagem cujo objetivo é a divulgação da saúde e consequentemente a manutenção da mesma (SANTOS MC, et al., 2016).

Este relato diz respeito às atividades desenvolvidas no projeto de extensão "Parasitoses na escola: promoção e prevenção à saúde em escolas do ensino fundamental em uma cidade no interior do Pará". O projeto visou promover a educação em saúde na prevenção de parasitoses entre crianças do ensino fundamental.

\section{RELATO DE EXPERIÊNCIA}

As atividades do projeto foram realizadas em uma escola de ensino fundamental com alunos da $3^{\text {a }}$ a $5^{\text {a }}$ séries de turnos matutino e vespertino no período de março a novembro de 2019. A equipe do trabalho foi composta por dois estudantes de graduação do curso de medicina pela coordenadora do projeto de extensão e docente da faculdade de. Junto com a equipe também a professora de cada turma e a diretora que acompanhavam as atividades junto com o grupo.

Ao chegarem na sala os membros da equipe se apresentavam para as crianças e explicavam o que estavam fazendo ali. Em seguida uma roda de conversa era iniciada. Nesse momento algumas perguntas eram feitas para as crianças sobre higiene pessoal, de alimentos e relacionadas ao possível conhecimento popular sobre parasitoses que poderiam ter.

Durante a interação pudemos avaliar que as crianças tinham conhecimento de que a água para ser consumida precisa ser tratada, embora não soubessem como isso era feito; assim como os alimentos e demais objetos que possam ser compartilhados devem ser previamente higienizados, ou, nas palavras deles: lavados.

Quando perguntamos sobre a escovação dos dentes percebemos que sabiam que ela deve ser feita todos os dias. Quanto a lavagem das mãos muitas crianças demonstraram saber a importância de fazê-lo pois eram orientadas por seus familiares a não permanecerem com elas sujas "pois fazia mal" ou "podia dar dor de barriga" (o que ficou claro para nós que estavam se referindo a questão de levar as mãos até a boca).

Também observamos que tinham conhecimento da existência de parasitos e/ou doenças por seus nomes mais populares tais como: lombriga e solitária, amarelão e barriga d'água, doença do jeca. Além disso 
compreendiam que a diarreia é um sintoma desses tipos de doenças pois alguns parentes ou eles mesmos já haviam adoecido e "o médico do postinho disse que era verme" e que, portanto, tinham que tomar "remédio pra verme".

Em seguida a roda de conversa um vídeo educativo chamado "Super Sabão contra as parasitoses" era apresentado para as crianças. O vídeo abrange de forma bastante lúdica as parasitoses mais comuns além de falar sobre higiene pessoal e coletiva. Em seguida, uma breve, clara e simples apresentação em Power Point era exibida com imagens dos principais parasitos de importância médica onde era abordado os seus ciclos de vida, sintomas, formas de transmissão e prevenção. A questão da vermifugação periódica também era salientada.

Também era dialogado com as crianças sobre a pele pode ser um reservatório de diversos microrganismos que podem ser transferidos de uma superfície para outra por meio de contato direto (pele com pele) ou indireto (pele com superfícies, equipamentos, objetos contaminados) sendo as mãos a principal via de transmissão desses microrganismos e que portanto a limpeza das mãos é muito importante e um meio eficaz de prevenção à doenças infecciosas, tais como as parasitoses visto que: a higienização das mãos consiste na remoção de sujidade, suor, oleosidade e células descamativas visando interromper a transmissão de microrganismos e é a medida individual mais simples e mais barata de prevenir a propagação de infecções relacionadas à saúde. Pode ser feita com uso de água e sabão, mas também com uso de álcool em gel, conforme indica o Ministério da Saúde (SILVA JUNIOR AF, et al., 2020).

Finalizada a apresentação era o momento de fazermos uma dinâmica com as crianças que consistia na demonstração do uso correto do álcool gel para limpeza das mãos. Inicialmente era informado que a lavagem das mãos embora muito importante para a descontaminação, muitas vezes não tem como ser feita, pois nem sempre temos uma pia, água e sabão disponíveis, mas que isso não era problema pois a descontaminação poderia ser feita com o álcool gel.

Informamos que o álcool gel pode ser adquirido de forma muito simples em farmácias, supermercados e lojas de departamento como a Americanas (que muitos conheciam) e que cada um deles deveria sempre ter consigo um pequeno frasco para ser utilizado quando necessário, quando não houver água e sabão disponíveis, mas for necessário limpas as mãos. Seguindo na dinâmica colocamos nas mãos de cada uma das crianças uma quantidade suficiente de álcool gel para uma desinfecção eficiente. Em seguida as crianças foram orientadas em como realizar a fricção antisséptica das mãos.

Importante salientar que o projeto foi iniciado em março de 2019. A previsão era de que pelo menos 3 escolas fossem visitadas com as atividades, mas tivemos dificuldades nos contatos com a direção das instituições de ensino além de imprevistos com semanas de provas e/ou demais atividades já programadas para as crianças.

Sendo assim, optamos em permanecer somente em uma escola, desde que todas as turmas do terceiro ao quinto anos fossem atendidas, em ambos os turnos, matutino e vespertino, almejando assim alcançar um número maior de crianças.

O público-alvo atendido foi de 240 crianças e o estudo observacional. Uma característica peculiar dessa escola de ensino é o seu serviço de inclusão, há um número significativo de crianças atendidas em condição especial e todas participaram das atividades.

\section{DISCUSSÃO}

Crianças são um grupo de indivíduos que frequentemente são acometidos por parasitoses principalmente quando iniciam suas vidas escolares. Partindo desse entendimento acreditamos ser muito importante que elas sejam orientadas de forma adequada a fim de que possam difundir o que aprendem na escola até sua família enfraquecendo dessa forma uma possível cadeia de transmissão de parasitos.

De acordo com Santos MC, et al. (2016), o ensino de parasitologia é de grande importância e deve ser abordado em sala de aula visando a prevenção das doenças causadas por parasitos a partir da educação em saúde, aqui entendida como um processo de ensino e aprendizagem cujo objetivo é a divulgação da saúde e consequentemente a prevenção e manutenção da mesma. 
É de suma importância que o ensino de ciências e/ou biologia se aproxime da realidade dos estudantes para que eles possam compreender a necessidade de estabelecer mudanças em seus hábitos de vida que vão favorecer a sua saúde e dos que Ihe cercam. De acordo com Silva PMC, et al. (2013), estudos demonstram que a utilização da ludicidade para abordar aspectos profiláticos de saúde contribui para a diminuição da propagação de doenças tendo em vista que estratégias educativas através da promoção e prevenção determinam melhoria no processo educativo já que acabam para atingir não só as crianças, mas também suas famílias.

A partir do momento que se trabalha com a transversalidade usando a educação em saúde torna-se possível oferecer possibilidades para o público-alvo poder tomar consciência das ações em detrimento de sua saúde e assim agir autonomamente em seu benefício próprio. Especificamente no que diz respeito as crianças da educação infantil, quando se trabalha utilizando abordagens com linguagens corporal e prática há a possibilidade do estabelecimento de uma relação mais significativa no cotidiano da aprendizagem (BARCELOS ISC, et al., 2019).

Para Tavares JS e Rodrigues WFG (2017), a escola é um espaço onde saúde e educação convergem permitindo uma ampla possibilidade de iniciativas a fim de incentivar as pessoas a alcançar o mais alto nível de bem-estar, de tal modo que possam desfrutar a vida o mais saudável possível e evitar doenças.

A escola é um espaço adequado para o desenvolvimento do pensamento crítico e político que contribui para a construção de valores pessoais, crenças e conceitos acerca do mundo e ajuda na produção social da saúde já que dessa forma a educação em saúde pode ser pautada nas histórias individuais se estendendo para professores, alunos, pais, mães avós e demais sujeitos inseridos nesse cenário, pois os programas de saúde fornecem serviços para os estudantes mas também servem à comunidade (BRASIL, 2011; SMELTZER SC e BARE BG, 2012).

A Organização Mundial da Saúde (OMS) recomenda a participação popular no controle de parasitoses de forma dinâmica e consciente, porém a falta de políticas públicas que atuem na prevenção e controle de doenças contribuiu para a alta disseminação nas camadas mais desfavorecidas da sociedade (NOVAES AKB, et al., 2017).

O Programa Saúde na Escola (PSE) foi instituído em 2007 na intenção de promover à comunidade escolar a participação em programas e projetos que articulem saúde e educação para o enfrentamento de vulnerabilidades que possam comprometer o pleno desenvolvimento de crianças e adolescentes. Contudo, assim como Programa Estratégia Saúde da Família (PSE) apresenta inúmeras falhas em sua execução e muitas vezes não cumpre com seu devido papel (BRASIL, 2011).

A escola onde foi realizada as atividades faz parte da rede de atendimento de uma unidade de saúde onde os discentes de medicina realizam suas atividades do curso. A partir da convivência com a equipe de saúde eles observaram que poderia ser importante a realização dessa ação. $O$ contato direto com as crianças que foram atendidas possibilitou uma visão mais consciente do ensino público o qual muitas vezes não permite ao professor condições favoráveis para função e tampouco permite abordar a saúde em sala de aula.

Segundo Barcelos ISC, et al. (2019), a educação em saúde é uma ferramenta importante no processo de aprendizagem de crianças e jovens pois visa a prevenção de inúmeros agravos e a extensão universitária permite que a universidade chegue mais próximo da comunidade podendo então realizar seu papel de prestar serviços à população além de ajudar na formação de profissionais com mais responsabilidade social e construção de indivíduos mais cientes de seus direitos. Acreditamos ser necessário a expansão para que outras escolas sejam assistidas a fim de atingir um contingente maior de infantes para assim poder ajudar a população no que diz respeito às parasitoses intestinais e colaborando com o trabalho dos professores nas escolas.

\section{REFERÊNCIAS}

1. BARCELOS ISC, et al. Ações de educação em saúde sobre parasitoses humanas em escolas públicas no município de Jataí, Goiás. Em Extensão, Uberlândia, 2019; 18(2): 133-141. 
2. BELO VS, et al. Fatores associados à ocorrência de parasitoses intestinais em uma população de crianças e adolescentes. Ver Paul Pediatr 2012;30(2):195-201.

3. BRAGAGNOLLO GR, et al. Intervenção educativa lúdica sobre parasitoses intestinais com escolares. Rev Bras Enferm [Internet]. 2019;72(5):1268-75.

4. BRASIL. Ministério da Saúde. Secretaria de Atenção à Saúde. Departamento de Atenção Básica. Instrutivo PSE/ Ministério da Saúde. Secretaria de Atenção à Saúde. Departamento de Atenção Básica. - Brasília: Ministério da Saúde, 2011.

5. DIAS DS, et al. Fatores de risco que contribuem para as parasitoses intestinais em crianças de 0 a 5 anos em Macapá - Amapá, Brasil. Ciência Equatorial, 2013; 3(1): 17-28.

6. HANEMANN T, et al. Parasitoses intestinais em crianças de creche/escolas de Porto Alegre: prevalência e profilaxia. Raízes e Rumos, 2015; 3(1): 182-183.

7. MAIA CVA, et al. Parasitoses intestinais em usuários do SUS em Limoeiro do Norte, Ceará, antes da expansão de sistema de esgotamento sanitário. HOLOS, 2015; 31(2): 98-109

8. MONTEIRO AMC, et al. Parasitoses intestinais em crianças de creches públicas localizadas em bairros periféricos do município de Coari, Amazonas, Brasil. Revista de Patologia Tropical, 2009; 38(4):284-290.

9. NESTI MMM, GOLDBAUM M. As creches e pré-escolas e as doenças transmissíveis. Jornal de Pediatria,2007; 83(4): 299-312.

10. SANTOS MC, et al. Ensino de parasitologia com crianças do ensino fundamental: utilização de modelos didáticos com massinha. Revista Fasem Ciências, 2016; 9(1): 05-15

11. SILVA JUNIOR AF, et al. Higienize Suas Mãos: Profissional Empoderado = Paciente Seguro. Altamira,2020;21p.

12. SILVA NETO AP, et al. Ação e prevenção: uma avaliação parasitológica em manipuladores de alimentos e escolares. Revista Extendere, 2013; 2(1): 23-35.

13. SILVA PMC, et al. Parasitoses intestinais: uma abordagem lúdica numa escola pública do município de Duque de Caxias, RJ. Saúde \& Ambiente em revista, 2013; 8(1): 43-53.

14. SMELTZER SC, BARE BG. Brunner \& Studart: Tratado de enfermagem médico-cirúrgica.12 ed. Rio de Janeiro: Guanabara Koogan,2012;2404p.

15. SOUSA FCA, et al. Prevalência de Parasitoses intestinais em crianças de uma escola pública municipal. REVI

16. TAVARES JS, RODRIGUES WFG. Promoção de educação em saúde para a prevenção de parasitoses: relato de experiência. Revista de Enfermagem UFPE online, 2017; 11(8): 3167-3170. 